\title{
Gastrointestinal well-being in subjects reporting mild gastrointestinal discomfort: characteristics and properties of a global assessment measure
}

\author{
Denis Guyonnet ${ }^{1 *}$, Bruce Naliboff ${ }^{2}$, Pascale Rondeau ${ }^{1}$, Emeran Mayer $^{2}$ and Olivier Chassany ${ }^{3}$ \\ ${ }^{1}$ Danone Research, RD128, 91767 Palaiseau, France \\ ${ }^{2}$ Gail and Gerald Oppenheimer Family, Center for Neurobiology of Stress, Division of Digestive Diseases, David Geffen School \\ of Medicine, UCLA, Los Angeles, CA, USA \\ ${ }^{3}$ Clinical Research Department, Assistance-Publique Hôpitaux E Paris-Diderot University, Paris, France \\ (Submitted 19 September 2012 - Final revision received 21 December 2012 - Accepted 11 January 2013 - First published online 22 February 2013)
}

\section{Abstract}

There is a lack of recognised markers for measuring gastrointestinal (GI) well-being and digestive symptoms in the general population. The aim of the present study was to demonstrate construct validity of a global assessment tool of GI well-being. In this randomised double-blind study, 197 adult women consumed either a probiotic fermented milk or a control dairy product daily during 4 weeks. GI well-being was assessed weekly using a single question and subjects indicated whether their GI well-being remained the same, improved or worsened compared with the baseline period. Responders for GI well-being were subjects reporting improvement for at least 2 weeks of the 4 weeks of intervention. Frequency of individual digestive symptoms was assessed weekly. Health-related quality of life (HRQOL) was measured at baseline and at the end of the study. Subjects reporting improvement of their GI well-being had a significantly $(P<0.05)$ lower frequency of combined digestive symptoms than individuals with no change, whereas subjects with worsened GI well-being had a significantly $(P<0 \cdot 05)$ higher digestive symptom frequency. Number of weeks with reported GI well-being improvement was significantly $(P<0.05)$ correlated with the decrease in digestive symptoms $(r 0.58)$ and the HRQoL digestive comfort dimension ( $r$ 0.47). When compared with non-responders, responders had significantly $(P<0.0001)$ fewer average digestive symptoms and higher scores on digestive comfort of the HRQOL questionnaire. The data provide construct validity for a single-item questionnaire as a measure of GI well-being improvement. These data support the use of this questionnaire as an end point for nutritional intervention in the general population.

\section{Key words: Gastrointestinal well-being: Patient-reported outcomes: Overall assessment}

Maintaining a healthy lifestyle including a healthy diet is important to support good health in humans. Digestive functions play a key role in maintaining or improving health status. In its guidance on health claims related to gut and immune function, the European Food and Safety Agency indicates that reducing gastrointestinal (GI) discomfort is considered as an indicator of improved GI function, and is a beneficial physiological effect ${ }^{(1)}$. The efficacy of probiotics or prebiotics/fibres on GI discomfort or well-being has been assessed during the last decade primarily in studies of patients with irritable bowel syndrome (IBS) ${ }^{(2-4)}$. In the absence of validated biomarkers, patient-reported outcomes (PRO) based on the patient's self-report are the standard used to determine the efficacy of these products on GI discomfort as well as on digestive symptoms.

The primary population targeted for dietary change and potentially beneficial food products is the general population, and, consequently, this population is a good and relevant study group for efficacy trials aimed at changing GI discomfort or well-being. Designing such trials is challenging because almost all clinical trials to date have been performed in IBS patients defined according to the Rome criteria ${ }^{(5)}$. Patients with IBS have generally been considered as an appropriate study group to substantiate claims on GI discomfort intended for the general population ${ }^{(1)}$. Although some digestive symptoms as well as alterations of bowel function are common in the general population ${ }^{(6-10)}$, there are significant differences in the type, severity and presentation of GI problems between those with IBS and the general population. For example, while IBS is characterised by abdominal pain and/or discomfort, digestive symptoms in the general population are less frequent and severe, and rarely include pain. On the other hand, predominant symptoms in non-patients include non-painful symptoms such as bloating, flatulence and borborygmi ${ }^{(6,7)}$.

Abbreviations: FBA, Food and Benefits Assessment; GI, gastrointestinal; HRQoL, Health-related quality of life; IBS, irritable bowel syndrome; PGWBI, Psychological General Well-Being Index; PRO, patient-reported outcome.

*Corresponding author: D. Guyonnet, fax +33169357696, email denis.guyonnet@danone.com 
Given these differences, it is unlikely that a PRO developed for the assessment of symptom improvement in IBS is appropriate for the assessment of improvement in mild GI discomfort and of GI well-being improvement in the general population. However, valid PRO are needed given the growing interest in testing products for the general population targeting GI discomfort or wellness.

In the general population with less frequent and less severe symptoms, the target of a food intervention is to improve the overall sensation of GI comfort or well-being. An overall assessment may allow individuals to use their own weighting of the importance of various aspects of GI comfort or wellbeing in determining the degree of change (improved, unchanged or worse). Due to the absence of a predominant symptom in this broad population, we hypothesised that people within the general population are more able to summarise adequately their symptom experience in a global single item than IBS patients, making the content validity of the global assessment more likely. A PRO measuring this concept must capture both improvement and worsening to ensure the absence of side effects of the food intervention that may occur with some fibres, for example. Overall binary assessment (e.g. adequate relief with yes/no modalities) that was extensively used in IBS trials was criticised for the absence of measuring worsening ${ }^{(11)}$.

There are no specific guidelines for developing PRO for foodrelated studies. In the absence of available valid and reliable PRO for measuring GI well-being or comfort in the general population, an overall assessment of GI well-being was developed using a three-point scale (improved, unchanged or worse $)^{(12)}$. This instrument was shown to be able to capture the beneficial effect of dairy products with probiotics in the non-IBS general population ${ }^{(12)}$.

In the present study, we explored the properties of this PRO end point in a non-IBS general population and compared changes in GI well-being with the change in a composite score of frequency of digestive symptoms, bowel function and health-related quality of life (HRQOL).

\section{Materials and methods}

\section{Study protocol and study subjects}

The study was single-centre (Harrison Clinical Centre, Munich, Germany), randomised, double-blind, controlled, parallelgroup design assessing the effect of a 4-week daily consumption of a fermented dairy product with probiotic $v$. a non-fermented dairy product with no probiotic (control group).

The study design, and inclusion and exclusion criteria of subjects have been described in detail in Guyonnet et al. ${ }^{(12)}$. All subjects were women, aged 18-60 years, with normal weight or overweight (BMI $18-30 \mathrm{~kg} / \mathrm{m}^{2}$ ) without a diagnosis of any digestive disease or systematic disease. Patients with IBS or any functional GI disorder were excluded as well as individuals under medications for digestive symptoms or with lactose intolerance. Subjects who took antibiotics within the month before the inclusion visit were not included.
Subjects must have bowel frequency within the normal range (3-21 bowel movements per week).

During a 2-week baseline period, baseline values were obtained for the outcome parameters with a weekly assessment of frequency of digestive symptoms (abdominal pain/discomfort, bloating, flatulence/passage of gas and borborygmi/rumbling stomach), bowel function (bowel movement and stool consistency) and HRQoL. Only subjects having a mean composite score for the frequency of digestive symptoms between 2 and 12 (score ranging from 0 (none of the four symptoms) to 16 (all symptoms every day)) during this baseline period were randomised. Subjects had bowel movement frequency within the normal range (3-21 per week). This allowed recruiting subjects among the general population experiencing a minimal level of digestive symptoms and who could benefit from the food intervention.

Randomised subjects consumed either two $125 \mathrm{~g}$ servings of a fermented dairy product with probiotic or a control dairy product daily during 4 weeks. The fermented dairy product with probiotic contained Bifidobacterium lactis (strain no. I-2494 in the French National Collection of Cultures of Microorganisms (CNCM, Paris, France)), referred to as DN-173 010 in previous publications such as in the Guyonnet et al. ${ }^{(12)}$ paper, together with the two classical yogurt starters, Streptococcus thermophilus (CNCM strain no. I-1630) and Lactobacillus delbrueckii subsp. bulgaricus (CNCM strain no. I-1632 and I-1519), and Lactococcus lactis ssp. lactis (CNCM strain no. I-1631). The test product contains $1.25 \times 10^{10}$ colony-forming units/cup of B. lactis CNCM I-2494/DN-173 010 and $1.2 \times 10^{9}$ colony-forming units/cup of $S$. thermophilus and Lactobacillus delbrueckii subsp. bulgaricus. The control dairy product was a milk-based non-fermented dairy product without probiotics and with a lactose content of $<4 \mathrm{~g} / \mathrm{cup}$, which is similar to the content of lactose in the test product. These two products had a similar appearance, texture and taste.

Subjects were not allowed to consume any probiotic food or supplement and fermented dairy product other than those provided for the intervention period.

The study protocol was conducted in accordance with the Declaration of Helsinki and approved by the Ethics Committee Bayerische Landesaerztekammer (Munich, Germany). All volunteers gave written informed consent before inclusion in the study.

\section{Overall gastrointestinal well-being}

GI well-being was self-evaluated by subjects weekly during the 4 weeks of product consumption. This scale was adapted from a scale developed by Guyatt et $a l^{(13)}$. Subjects assessed the changes in their GI well-being with the following question: "How do you consider in the past $7 \mathrm{~d}$, your GI well-being (intestinal transit, stool frequency and consistency, abdominal pain/discomfort, bloating, flatulence/passage of gas, borborygmi/rumbling stomach) compared to the period before beginning the consumption of the study product?'. In order to have a simple instrument and decrease ambiguity in the subject's assessment, a three-point Likert scale was used to report changes (improved, unchanged or worse) in GI well-being. The fifteen-point answer option of the original 
Guyatt global assessment was considered as inappropriate to a population with mild digestive symptoms, i.e. seven-point $(-7$ to -1$)$ for subjects answering 'worse' and seven-point $(+1$ to +7$)$ for subjects answering 'improved'. Specifically, the concern was that subjects with and infrequent symptoms would not be able to reliably discriminate GI well-being using such a fine-grained scale.

Overall GI well-being improvement for the study period was calculated as the number of weeks rated as 'improved' during the 4-week period of intervention (range 0-4).

As recommended in guidelines for the design of treatment trials for functional GI disorders ${ }^{(14)}$, each subject was classified as a responder or a non-responder for GI well-being that was used as the primary end point in the intervention trial $^{(12)}$. The definition of a responder must reflect a clinically meaningful improvement for each participant. In the absence of consensus on what constitutes a clinically meaningful improvement, the pre-specified definition of a responder was based on the recommendations for the global assessment of symptom relief in IBS trials ${ }^{(14)}$.

A responder was defined as a subject having an improvement in their GI well-being, i.e. answering 'improved' on the three-point Likert scale, on at least 2 weeks over the 4 -week period of product consumption. Recent guidelines for defining responders for abdominal pain in IBS suggest possible use of a 'no worsening' condition ${ }^{(15)}$. In the present analysis, an alternative responder definition, another definition of a responder including no rating of 'worsening' at any week during the 4-week period of product consumption, was therefore used to perform exploratory sensitivity analysis.

\section{Digestive symptoms}

The frequency of four individual digestive symptoms (abdominal pain/discomfort, bloating, flatulence/passage of gas and borborygmi/rumbling stomach) was evaluated weekly with five-point Likert scales that range from 0 (never) to 4 (every day of the week) throughout the study. A composite score of all symptoms was calculated ranging from 0 to 16 .

\section{Stool frequency and consistency}

Bowel movements were reported daily throughout the study as well as stool consistency for each stool passed according to the Bristol Stool Form Scale ${ }^{(16)}$. In order to assess the normalisation of stool consistency, scores of stool consistency were recoded as follow: $0=$ type 4 (like a sausage or snake, smooth and soft); $1=$ types 3 (like a sausage but with cracks on surface) and 5 (soft blobs with clear-cut edges); 2 types 2 (sausage shaped but lumpy) and 6 (fluffy pieces with ragged edges, a mushy stool); $3=$ types 1 (separate hard lumps like nuts, difficult to pass) and 7 (watery, no solid pieces, entirely liquid).

\section{Health-related quality of life questionnaires}

HRQOL of subjects was assessed by self-administration of two questionnaires: the Food and Benefits Assessment
$(\mathrm{FBA})^{(17)}$ and the Psychological General Well-Being Index (PGWBI) ${ }^{(18)}$. The questionnaires were completed at baseline and after 4 weeks of product consumption.

The FBA questionnaire comprises forty-one items, making it possible to calculate scores for seven dimensions (snacking, vitality, well-being, physical appearance, aesthetics, digestive comfort and disease prevention). The scores range from 0 to 100 (best).

The generic questionnaire PGWBI measures psychological well-being and distress, and is composed of twenty-two items that constitute six dimensions (anxiety, depression, self-control, positive well-being, general health and vitality). The scores of all dimensions can be summarised to provide a global score ${ }^{(18)}$. The scores range from 0 to 100 (best).

The FBA digestive comfort dimension score and the PGWBI global score were defined as the main scores for HRQOL analysis. Other dimensions of both questionnaires were considered as secondary HRQoL criteria.

\section{Statistical analyses}

All the analyses were carried out on the intention-to-treat population. At baseline, descriptive statistics were done (mean, standard deviation and CI with a risk fixed at 5\%) on digestive symptoms, composite score, stool frequency and consistency, digestive dimension of the FBA questionnaire and the PGWBI, age and BMI.

The change from baseline, at each week, was calculated for digestive symptoms, composite score, stool consistency and frequency.

For each week and by the degree of GI well-being (improved, unchanged or worse), the mean change of each previous outcome was calculated.

By week and over the 4 weeks of product consumption, a comparison of the mean change of the outcomes was carried out between degrees of GI well-being. An ANOVA followed by a Tukey-Kramer adjustment test, to take into account the multiplicity of tests, was used to test for statistical differences.

For each subject, the number of weeks rated as 'improved' for overall GI well-being during the 4-week period of intervention was counted, allowing classification of subjects in five groups corresponding to $0,1,2,3$ or 4 weeks with 'improved' overall GI well-being. For each group, the mean and standard deviation of digestive symptom, composite score, the four individual digestive symptoms, stool consistency and frequency, and HRQoL responses were calculated at baseline. The correlation between the number of weeks of improvements and the other outcomes was evaluated with a Spearman correlation coefficient (non-parametric correlation test).

A similar analysis was carried out on the mean change from baseline of the different outcomes and the number of weeks of GI well-being improvement over the 4 weeks ( $0-4$ weeks) and the overall GI well-being responder status (yes/no).

In addition to the aforementioned primary analyses, two exploratory analyses were also performed to further examine potential clinical utility of the instrument.

The first one consists to cross the overall GI well-being responder status and digestive symptom responder status. 
Table 3. Mean values and correlations between the number of weeks with improved gastrointestinal (GI) well-being and the average change in the other outcome measures

(Mean values and standard deviations, $n$ 197)

\begin{tabular}{|c|c|c|c|c|c|c|c|c|c|c|c|}
\hline & \multirow[b]{2}{*}{$n$} & \multicolumn{2}{|c|}{$\begin{array}{l}\text { Digestive symp- } \\
\text { tom composite } \\
\text { score }\end{array}$} & \multicolumn{2}{|c|}{$\begin{array}{c}\text { Stool } \\
\text { consistency } \dagger\end{array}$} & \multicolumn{2}{|c|}{$\begin{array}{c}\text { Stool } \\
\text { frequency }\end{array}$} & \multicolumn{2}{|c|}{$\begin{array}{l}\text { FBA - diges- } \\
\text { tive comfort† }\end{array}$} & \multicolumn{2}{|c|}{ PGWBI† } \\
\hline & & Mean & SD & Mean & SD & Mean & SD & Mean & SD & Mean & SD \\
\hline \multicolumn{12}{|c|}{ Improved GI well-being } \\
\hline 0 week & 63 & -0.19 & 1.59 & -0.07 & 0.43 & 0.24 & 1.42 & 2.51 & 11.4 & $3 \cdot 61$ & 8.45 \\
\hline 1 week & 47 & -1.35 & $2 \cdot 15$ & -0.12 & 0.36 & 1.40 & 1.77 & $6 \cdot 56$ & 11.6 & 3.81 & 9.55 \\
\hline 2 weeks & 37 & $-2 \cdot 20$ & $1 \cdot 70$ & -0.21 & 0.36 & $1 \cdot 11$ & $2 \cdot 04$ & $10 \cdot 1$ & $12 \cdot 0$ & $3 \cdot 17$ & $7 \cdot 87$ \\
\hline 3 weeks & 33 & -3.04 & 1.91 & -0.45 & 0.49 & 1.7 & 2.35 & $17 \cdot 3$ & $10 \cdot 7$ & $6 \cdot 96$ & $10 \cdot 74$ \\
\hline 4 weeks & 17 & -4.04 & $2 \cdot 40$ & -0.44 & 0.50 & $1 \cdot 21$ & 3.38 & $20 \cdot 6$ & $16 \cdot 1$ & 4.22 & $12 \cdot 21$ \\
\hline Correlations & & \multicolumn{2}{|c|}{$-0.58^{\star \star \star}$} & \multicolumn{2}{|c|}{$-0.28^{\star \star \star}$} & \multicolumn{2}{|c|}{$0.25^{\star \star}$} & \multicolumn{2}{|c|}{$0.46^{\star \star \star}$} & \multicolumn{2}{|c|}{$0 \cdot 10$} \\
\hline
\end{tabular}

FBA, Food and Benefits Assessment; PGWBI, Psychological General Well-Being Index.

Correlations between the number of weeks of Gl well-being improvement and the other parameters were significant (Spearman's non-parametric test): ${ }^{\star \star} P<0.001$, ${ }^{\star \star \star} P<0.0001$.

† Outcome measure changes are calculated using the mean change over the 4-week period of intervention $v$. baseline values.

$\ddagger$ Number of weeks with improved Gl well-being during the 4 weeks of intervention.

outcomes are shown in Table 2. Regardless of the time point in the study, subjects reporting improved GI well-being had significantly larger decreases in the composite score of digestive symptoms than subjects with unchanged or worse GI well-being.

The unchanged group had also decreases in composite score, whereas the worsened group experienced increased composite score, these two groups also being significantly different.

For stool frequency and consistency, differences were only found between those rating their GI well-being as improved and worsened at week 2 for consistency and at week 4 for both consistency and frequency.

There was a significant $(P<0.0001)$ correlation between the number of weeks of GI well-being improvement and mean changes in the composite score of digestive symptoms $(-0.58)$ and scores on the digestive comfort dimension of the FBA questionnaire $(-0 \cdot 46)$ (Table 3$)$. Correlations with stool consistency and frequency were also significant, although modest $(-0.28$ and $0 \cdot 25$, respectively). The mean changes over the 4-week period of intervention for each individual digestive symptom were also significantly $(P<0 \cdot 0001)$ correlated with the number of weeks rated as 'improved' for GI well-being (Table 4). These correlations were all inferior to the correlation of the composite score, with flatulence showing the highest correlation $(-0.50)$

\section{Comparison of gastrointestinal well-being scores between responders and non-responders}

Subjects who were responders to GI well-being assessment when compared with non-responders had clearly better GI health status as shown by a significantly greater improvement in the composite score of digestive symptoms $(P<0.0001$, $-38.5 \%$ in the responder group $v .-9.9 \%$ in the non-responder group; Table 5). A significantly greater improvement in bowel function (stool frequency, $P<0.0001$ and consistency, $P<0.05$ ) was also observed for the responder group compared with non-responders as well as a greater improvement in HRQOL from the digestive comfort dimension of the FBA questionnaire $(P<0 \cdot 0001)$. Sensitivity analysis with a more strict definition of responders (i.e. without worsening at any week) showed similar data (decrease in digestive symptoms:

Table 4. Mean values and correlations between the number of weeks with improved gastrointestinal (Gl) well-being and the average change in individual digestive symptoms

(Mean values and standard deviations, $n$ 197)

\begin{tabular}{|c|c|c|c|c|c|c|c|c|c|}
\hline & \multirow[b]{2}{*}{$n$} & \multicolumn{2}{|c|}{$\begin{array}{l}\text { Abdominal pain/ } \\
\text { discomfort score }\end{array}$} & \multicolumn{2}{|c|}{$\begin{array}{l}\text { Bloating } \\
\text { scoret }\end{array}$} & \multicolumn{2}{|c|}{ Flatulence score $†$} & \multicolumn{2}{|c|}{$\begin{array}{l}\text { Borborygmi } \\
\text { score }\end{array}$} \\
\hline & & Mean & SD & Mean & SD & Mean & SD & Mean & SD \\
\hline \multicolumn{10}{|c|}{ Improved GI well-beingł } \\
\hline 0 week & 63 & -0.04 & 0.53 & -0.14 & 0.62 & 0.04 & 0.52 & -0.04 & 0.77 \\
\hline 1 week & 47 & -0.26 & 0.71 & -0.49 & 0.83 & -0.24 & 0.81 & -0.32 & 0.70 \\
\hline 2 weeks & 37 & -0.34 & 0.47 & -0.86 & 0.62 & -0.59 & 0.78 & -0.40 & 0.69 \\
\hline 3 weeks & 33 & -0.66 & 0.65 & -0.83 & 0.56 & -0.78 & 0.77 & -0.77 & 0.69 \\
\hline 4 weeks & 17 & -0.84 & 0.87 & $-1 \cdot 10$ & 1.04 & $-1 \cdot 21$ & 0.88 & -0.90 & 0.75 \\
\hline Correlations & & \multicolumn{2}{|c|}{$-0.38^{\star \star \star}$} & \multicolumn{2}{|c|}{$-0.42^{\star \star \star}$} & \multicolumn{2}{|c|}{$-0.50^{\star \star \star}$} & \multicolumn{2}{|c|}{$-0.39^{\star \star \star}$} \\
\hline
\end{tabular}

Correlations between the number of weeks of Gl well-being improvement and the other parameters were significant (Spearman's non-parametric test): ${ }_{\star \star *} P<0.0001$.

† Outcome measure changes are calculated using the mean change over the 4-week period of intervention $v$. baseline values.

$\ddagger$ Number of weeks with improved GI well-being during the 4 weeks of intervention. 
Table 5. Comparison of outcome measures in gastrointestinal (Gl) well-being responders and non-responders

(Mean values and standard deviations)

\begin{tabular}{|c|c|c|c|c|c|c|c|c|c|c|c|}
\hline & \multirow[b]{2}{*}{$n$} & \multicolumn{2}{|c|}{$\begin{array}{l}\text { Digestive symp- } \\
\text { tom composite } \\
\text { score } \dagger\end{array}$} & \multicolumn{2}{|c|}{$\begin{array}{c}\text { Stool } \\
\text { consistency } \dagger\end{array}$} & \multicolumn{2}{|c|}{$\begin{array}{c}\text { Stool } \\
\text { frequency }\end{array}$} & \multicolumn{2}{|c|}{$\begin{array}{l}\text { FBA - digestive } \\
\text { comfort }\end{array}$} & \multicolumn{2}{|c|}{ PGWBI† } \\
\hline & & Mean & SD & Mean & SD & Mean & SD & Mean & SD & Mean & SD \\
\hline \multicolumn{12}{|c|}{ Responder GI well-being } \\
\hline Yes & 87 & $-2 \cdot 88^{\star \star}$ & 2.03 & $-0.34^{\star \star}$ & 0.45 & $1 \cdot 38^{*}$ & 2.45 & $14 \cdot 88^{\star \star}$ & 13.00 & $4 \cdot 82$ & 9.98 \\
\hline No & 110 & -0.68 & 1.93 & -0.09 & 0.40 & 0.74 & 1.67 & $4 \cdot 24$ & 11.61 & 3.69 & 8.90 \\
\hline
\end{tabular}

FBA, Food and Benefits Assessment; PGWBI, Psychological General Well-Being Index.

Mean values were significantly different compared with the non-responder group $\left(t\right.$ test): ${ }^{\star} P<0.05,{ }^{\star \star} P<0.0001$.

$\dagger$ Changes are calculated using the mean change over the 4-week period of intervention $v$. baseline values.

$\ddagger A$ responder is defined as a subject reporting an improvement of its GI well-being during at least 2 weeks of the 4 weeks of intervention.

$-41 \cdot 8 \%$ in responders $v$. $-10 \cdot 9 \%$ in non-responders), except

for stool frequency that was no longer significant (Table 6).

\section{Effect of baseline status on gastrointestinal well-being improvement and responder status}

Improvement in GI well-being during the 4 weeks of intervention was not correlated ( $r-0.09$ to $0 \cdot 17)$ with the baseline value of any outcome (composite score of digestive symptoms, stool frequency and consistency, and HRQOL).

Responder status was also evaluated for its relationship with baseline symptom measures. Scores for individual digestive symptoms and composite score were generally higher in responders $v$. non-responders (Table 7 ), with abdominal pain $(P=0.02)$ and stool consistency $(P=0.02)$ being the only symptoms statistically significantly higher in responders when compared with non-responders. Similar results were found with the alternative 'no worsening' definition of a responder with significant differences for abdominal pain $(P=0.03)$, stool consistency $(P=0.02)$ and the composite score $(P=0 \cdot 01)$ (Table 8$)$.

\section{Exploratory analyses}

The distribution of the two modalities (yes/no) was comparable for GI well-being responders and for the decrease in the composite score of digestive symptom responders (Fisher's exact test, $P<0.0001, \kappa=0 \cdot 44)$. The true positive rate (yes for both parameters) was $65 \cdot 1 \%$ (fifty-six/eighty-six responders for digestive symptoms) and the true negative rate (no for both parameters) is $79 \cdot 3 \%$ (eighty-four/106 nonresponders for digestive symptoms). The corresponding negative and positive predictive values for GI well-being are $73.7 \%$ (eighty-four/114 non-responders for GI well-being) and $71 \cdot 8 \%$ (fifty-six/seventy-eight responders for GI well-being), respectively.

The partial least square analysis investigating the contribution of the different symptoms (abdominal pain, bloating, flatulence and borborygmi) and stool frequency and consistency to the improvement of GI well-being shows that the four symptoms are the major contributors. These symptoms accounted for $73 \cdot 2 \%$, whereas $26 \cdot 8 \%$ of the model was explained by stool consistency (21.2\%) and stool frequency (5.6\%). Among the digestive symptoms, flatulence had the higher impact (30.9\%) followed by bloating (16.9\%) and abdominal pain (15.8\%), with borborygmi having the lower impact ( $9 \cdot 6 \%)$.

\section{Discussion}

The present study provides preliminary construct validity for a single-item PRO as a measure of GI well-being in a non-IBS population, by demonstrating that this measure is reliably associated with a broad range of digestive symptom changes. These data support the use of this PRO as an end point for clinical trials in the general healthy population with mild GI non-painful discomfort, aiming at demonstrating the effect of specific foods on GI (dis)comfort and common GI symptoms.

In the absence of validated biomarkers, it is necessary that the primary outcome measure in studies targeting overall GI well-being or (dis)comfort be based on patient report, i.e. a

Table 6. Sensitivity analysis for comparison of outcome measures in gastrointestinal (GI) well-being responders and non-responders (Mean values and standard deviations)

\begin{tabular}{|c|c|c|c|c|c|c|c|c|c|c|c|}
\hline & \multirow[b]{2}{*}{$n$} & \multicolumn{2}{|c|}{$\begin{array}{l}\text { Digestive symp- } \\
\text { tom composite } \\
\text { score } \dagger\end{array}$} & \multicolumn{2}{|c|}{$\begin{array}{c}\text { Stool } \\
\text { consistency } \dagger\end{array}$} & \multicolumn{2}{|c|}{$\begin{array}{c}\text { Stool } \\
\text { frequency† }\end{array}$} & \multicolumn{2}{|c|}{$\begin{array}{l}\text { FBA - digestive } \\
\text { comfort† }\end{array}$} & \multicolumn{2}{|c|}{ PGWBI† } \\
\hline & & Mean & SD & Mean & SD & Mean & SD & Mean & SD & Mean & SD \\
\hline \multicolumn{12}{|c|}{ Responder GI well-beingf } \\
\hline Yes & 73 & $-3 \cdot 19^{\star \star}$ & 1.93 & $-0.36^{*}$ & 0.45 & 1.30 & $2 \cdot 50$ & $15 \cdot 41^{\star *}$ & 13.43 & 5.00 & $10 \cdot 24$ \\
\hline No & 124 & -0.75 & 1.92 & -0.11 & 0.41 & 0.85 & 1.77 & $5 \cdot 13$ & 11.73 & 3.72 & 8.86 \\
\hline
\end{tabular}

FBA, Food and Benefits Assessment; PGWBI, Psychological General Well-Being Index.

Mean values were significantly different compared with the non-responder group $\left(t\right.$ test): ${ }^{\star} P<0.05,{ }^{*} P<0.0001$.

$\dagger$ Changes are calculated using the mean change over the 4-week period of intervention $v$. baseline values.

$\ddagger A$ responder is defined as a subject reporting an improvement of its Gl well-being during at least 2 weeks of the 4 weeks of intervention without worsening at any time. 
Table 7. Baseline characteristics of gastrointestinal (GI) well-being responders and non-responders (Mean values and standard deviations, $n$ 197)

\begin{tabular}{|c|c|c|c|c|c|}
\hline & \multicolumn{2}{|c|}{$\begin{array}{l}\text { Non-responders } \\
\quad(n 110)\end{array}$} & \multicolumn{2}{|c|}{$\begin{array}{l}\text { Responders } \\
(n 87)^{\star}\end{array}$} & \multirow[b]{2}{*}{$P$} \\
\hline & Mean & SD & Mean & SD & \\
\hline Abdominal pain/discomfort & 0.91 & 0.71 & $1 \cdot 16$ & 0.77 & 0.02 \\
\hline Bloatingt & 1.99 & 0.84 & $2 \cdot 14$ & 0.78 & 0.26 \\
\hline Flatulence $†$ & $2 \cdot 40$ & 0.83 & 2.59 & 0.85 & 0.08 \\
\hline Borborygmit & 1.57 & 0.95 & 1.59 & 0.84 & 0.80 \\
\hline Digestive symptom composite scoreł & 6.86 & $2 \cdot 29$ & 7.48 & 1.99 & 0.05 \\
\hline Stool frequency & $7 \cdot 12$ & 2.99 & 7.49 & 3.05 & 0.35 \\
\hline Stool consistency§ & 1.21 & 0.55 & 1.40 & 0.53 & 0.02 \\
\hline FBA - digestive comfort $\|$ & $66 \cdot 64$ & $12 \cdot 63$ & 63.73 & 13.09 & 0.17 \\
\hline PGWBI\| & 73.65 & 11.93 & $72 \cdot 72$ & 11.51 & 0.54 \\
\hline
\end{tabular}

FBA, Food and Benefits Assessment; PGWBI, Psychological General Well-Being Index.

${ }^{*} A$ responder is defined as a subject reporting an improvement of its GI well-being during at least 2 weeks of the 4 weeks of intervention.

†Frequency of symptom, score 0 (never) to 4 (every day of the week). Values represent the two weekly assessments during baseline.

‡ Adapted from the Bristol Stool Form Scale: 0 (type 4); 1 (types 3 and 5); 2 (types 2 and 6); 3 (types 1 and 7).

§ Sum of individual symptoms, score $0-16$.

|l Score from 0 to 100 (best)

PRO. The use of a validated questionnaire is required to demonstrate a beneficial physiological effect of a food on GI discomfort for health claim in Europe ${ }^{(1)}$. Important research efforts have been made in the past 10 years to improve PRO used in IBS trials ${ }^{(15,19)}$, but despite the high interest in making a claim on GI discomfort for foods, there has been little emphasis to develop PRO to evaluate this construct in the general population. Developing an instrument measuring GI comfort or well-being outside the IBS population is challenging due to the absence of pain, and the lower level of GI discomfort and symptoms reported by healthy subjects. However, subjects among the general population frequently experience non-painful digestive symptoms ${ }^{(6-8)}$ and the development of food aiming at improving their GI heath status requires appropriate tools.
The results obtained in the present study demonstrate that the simple weekly global rating of change in GI well-being is a good representation of multidimensional change in digestive symptoms and is also significantly, but to a lesser extent, related to changes in bowel function. This is supported by the similar significant correlation values between each of the four digestive symptoms (abdominal pain/discomfort, bloating, borborygmi/rumbling stomach and flatulence) and the number of weeks with improved GI well-being. This single-item PRO seems to be able to capture important signs and symptoms that can have an impact of GI (dis)comfort, and to summarise adequately the mild digestive symptoms commonly reported by the general population. In contrast, such a single-item global assessment may not be suitable for clinical trials in the IBS population, where patients suffer

Table 8. Baseline characteristics of gastrointestinal (GI) well-being responders and non-responders with a modified definition

(Mean values and standard deviations, $n$ 197)

\begin{tabular}{|c|c|c|c|c|c|}
\hline & \multicolumn{2}{|c|}{$\begin{array}{l}\text { Non-responders } \\
\quad(n 124)\end{array}$} & \multicolumn{2}{|c|}{$\begin{array}{l}\text { Responders } \\
(n 73)^{*}\end{array}$} & \multirow[b]{2}{*}{$P$} \\
\hline & Mean & SD & Mean & SD & \\
\hline Abdominal pain/discomfort $†$ & 0.93 & 0.70 & $1 \cdot 17$ & 0.80 & 0.03 \\
\hline Bloatingt & 1.97 & 0.83 & $2 \cdot 19$ & 0.79 & 0.07 \\
\hline Flatulence $\dagger$ & 2.40 & 0.83 & $2 \cdot 62$ & 0.84 & 0.08 \\
\hline Borborygmi† & 1.54 & 0.95 & 1.64 & 0.82 & 0.30 \\
\hline Digestive symptom composite scoreł & $6 \cdot 84$ & $2 \cdot 23$ & $7 \cdot 63$ & 2.00 & 0.01 \\
\hline Stool frequency & 7.08 & 2.95 & 7.63 & $3 \cdot 11$ & 0.20 \\
\hline Stool consistency§ & 1.23 & 0.55 & 1.41 & 0.53 & 0.02 \\
\hline FBA - digestive comfort\| & $66 \cdot 51$ & $12 \cdot 74$ & 63.39 & 12.97 & $0 \cdot 13$ \\
\hline PGWBI\| & 73.37 & 12.09 & 73.04 & $11 \cdot 17$ & 0.74 \\
\hline
\end{tabular}

FBA, Food and Benefits Assessment; PGWBI, Psychological General Well-Being Index.

* $A$ responder is defined as a subject reporting an improvement of its Gl well-being during at least 2 weeks of the 4 weeks of intervention without worsening at any time.

†Frequency of symptom, score 0 (never) to 4 (every day of the week). Values represent the two weekly assessments during baseline.

‡Adapted from the Bristol Stool Form Scale: 0 (type 4); 1 (types 3 and 5); 2 (types 2 and 6); 3 (types 1 and 7 ). $\S$ Sum of individual symptoms, score $0-16$.

\|l Score from 0 to 100 (best). 
from a greater number and severity of symptoms, including abdominal pain, and treatments are more targeted for specific symptoms ${ }^{(11)}$.

This PRO is an improved construct compared with the binary outcome used in the past in IBS clinical trials that did not include an answer option capturing worsening of condition $^{(11,20)}$. At least for the composite measure of digestive symptoms, a rating of worse GI well-being was clearly associated with a negative change in digestive symptoms. Although valid assessment of worsening symptoms may not be relevant for measures of responder status in all trials, for some it may be important to separate out those with a positive or no change from those with worse outcomes. The present data clearly showed the limitations and weaknesses of the use of a binary response (improved or unchanged) that may mask potential negative effects.

Beyond the statistical significance, the nature and size of biological relevance of an effect is a key criterion when assessing the efficacy of a food/nutritional intervention ${ }^{(21)}$. When using PRO, a responder definition is usually needed to identify participants who achieve a predefined clinically meaningful improvement, and proportions of responders are then compared to determine the efficacy of the tested product. We used a similar definition of responders as that used extensively in IBS drug trials for binary outcome, i.e. improvement during at least $50 \%$ of the time. We show that the responder definition for GI well-being appears to be well correlated with changes in digestive symptoms and other parameters associated with GI health. Subjects classified as responders had a significantly higher decrease in digestive symptoms $(-38.5 \%)$ when compared with non-responders $(-9.9 \%)$. This was associated with improved bowel function and with a significantly higher score for a specific digestive comfort dimension of the HRQOL questionnaire. This responder definition has been shown to be responsive to a probiotic intervention in a double-blind, randomised, controlled trial ${ }^{(12)}$. An exploratory analysis using a more strict definition of responders (including no worsening at any time point) showed similar differences between responders and nonresponders with the same magnitude of symptom decrease $(-41.8 \%)$ in the responder group.

The present data highlight that changes in GI well-being in this non-IBS population are primarily related to changes in digestive symptoms. The composite score of digestive symptoms showed a higher value of correlation with improvement of GI well-being, and worsening of GI well-being is associated with an increase in digestive symptoms. However, this is not driven by a specific symptom such as abdominal pain/discomfort (as found in IBS), and all the four digestive symptoms measured in the present study contribute in the same way to the improvement of GI well-being. These data confirm the multidimensional nature of this concept.

It has been suggested that the impact of baseline digestive symptom severity is a confounding factor for the effect of treatment outcome in $\operatorname{IBS}^{(20,22)}$. We did not find a significant association between baseline digestive symptom severity and GI well-being improvement in the present study as well as with baseline data of bowel function and HRQoL. Even though responders reported the highest level of abdominal pain/discomfort, composite score of digestive symptoms and stool consistency, no significant correlation was found between all the outcomes and the level of GI well-being improvement.

It is important to point out that some of the findings may reflect specifics of the intervention study from which the data were collected. For example, the fact that digestive symptoms are more related to changes in GI well-being than bowel habit may be a result of greater change in symptoms in the present study $v$. bowel habit and not inherent in the measure itself. This population has normal bowel habits, and investigations in individuals with altered bowel functions may provide additional information on the factors that could have an impact on this single-item PRO for GI well-being. Data were obtained in a short-term study (4-week intervention) and a longer duration of intervention should allow determining the stability of the measure over a longer period as well as its relationship to changes of other parameters.

This overall assessment may be useful in any clinical trial assessing the effect of a food or nutritional intervention on GI comfort or discomfort, and is complementary to the traditional symptom assessment and bowel function parameters. The choice of the main outcome (overall assessment of GI well-being or digestive symptoms) in a specific clinical trial should be based on the expected effects of the intervention (e.g. what is the main biological/physiological effect of the intervention?). Overall assessment of GI well-being cannot be used alone as the main outcome because such overall improvement should be supported by a concomitant improvement of GI symptoms and/or bowel functions. Taking together, these data could allow us to understand the physiological effect of the intervention and its relevance for a well-defined target population. This overall assessment could be useful as a secondary outcome in trials looking at bowel functions as the main outcome. This may help in determining whether improved bowel functions are associated with GI comfort or discomfort improvement, as the real benefit for a subject for a significant increase of one stool per week could be questionable.

In summary, the overall assessment of GI well-being as used in the present study may be a useful PRO for use in the general non-IBS population which correlates well with changes in digestive symptoms. As such, it appears to be a relevant measure to assess the benefits of food intervention in a healthy population.

\section{Acknowledgements}

The present study was funded by Danone Research. The study was performed in one clinical centre (Harrison Clinical Centre, Munich, Germany). D. G., B. N. and O. C. were responsible for the study design and for the interpretation of the study results. P. R. performed the statistical analysis. E. M. was involved in the interpretation of the results. All the authors were involved in the writing and validation of the paper. D. G. and P. R. are employees of Danone Research. B. N., E. M. and O. C. are consultants of Danone Research. 


\section{References}

1. EFSA on Dietetic Products, Nutrition and Allergies (NDA) (2011) Guidance on the scientific requirements for health claims related to gut and immune function. EFSA J 9, 1984.

2. Clarke G, Cryan JF, Dinan TG, et al. (2012) Review article: probiotics for the treatment of irritable bowel syndrome focus on lactic acid bacteria. Aliment Pharmacol Ther 35 , 403-413.

3. Whelan K (2011) Probiotics and prebiotics in the management of irritable bowel syndrome: a review of recent clinical trials and systematic reviews. Curr Opin Clin Nutr Metab Care 14, 581-587.

4. Moayyedi P, Ford AC, Talley NJ, et al. (2010) The efficacy of probiotics in the treatment of irritable bowel syndrome: a systematic review. Gut 59, 325-332.

5. Longstreth GF, Thompson WG, Chey WD, et al. (2006) Functional bowel disorders. Gastroenterology 130, 1480-1491.

6. van Kerkhoven LA, Eikendal T, Laheij RJ, et al. (2008) Gastrointestinal symptoms are still common in a general Western population. Neth J Med 66, 18-22.

7. Leibbrand R, Cuntz U \& Hiller W (2002) Assessment of functional gastrointestinal disorders using the GastroQuestionnaire. Int J Behav Med 9, 155-172.

8. Frexinos J, Denis P, Allemand H, et al. (1998) Descriptive study of functional digestive symptoms in the French general population. Gastroenterol Clin Biol 22, 785-791.

9. Talley NJ, Boyce P \& Jones M (1998) Identification of distinct upper and lower gastrointestinal symptom groupings in an urban population. Gut 42, 690-695.

10. Heaton KW, O'Donnell LJD, Braddon FE, et al. (1992) Symptoms of irritable bowel syndrome in a British urban community: consulters and nonconsulters. Gastroenterology 102, 1962-1967.

11. Trentacosti AM, He R, Burke LB, et al. (2010) Evolution of clinical trials for irritable bowel syndrome: issues in end points and study design. Am J Gastroenterol 105, 731-735.

12. Guyonnet D, Schlumberger A, Mhamdi L, et al. (2009) Fermented milk containing Bifidobacterium lactis DN-173010 improves gastrointestinal well-being and digestive symptoms in a global population of women. A randomised, doubleblind, parallel, controlled study. Br J Nutr 102, 1654-1662.

13. Guyatt GH, Deyo RA, Charlson M, et al. (1989) Responsiveness and validity in health status measurement: a clarification. J Clin Epidemiol 42, 403-408.

14. Irvine EJ, Whitehead WE, Chey WD, et al. (2006) Design of treatment trials for functional gastrointestinal disorders. Gastroenterology 130, 1538-1551.

15. US Food and Drug Administration (2010) Guidance for industry irritable bowel syndrome - clinical evaluation of products for treatment. http://www.fda.gov/Drugs/ GuidanceComplianceRegulatoryInformation/Guidances/ default.htm

16. O'Donnell LJD, Virjee J \& Heaton KW (1990) Detection of pseudodiarrhoea by simple clinical assessment of intestinal transit rate. $\mathrm{Br}$ Med J 300, 439-440.

17. Guyonnet D, Chassany O, Picard C, et al. (2008) Perceived subject outcomes and impact on health-related quality of life associated with diet using the new Food Benefits Assessment (FBA) questionnaire: development and psychometric validation. Public Health Nutr 11, 1163-1172.

18. Dupuy HJ (1984) The psychological general well-being (PGWB) index. In Assessment of Quality of Life in Clinical Trials of Cardiovascular Therapies, chapter 9, pp. 170-184 [NK Wenger, ME Mattson, CF Furberg and J Elinson, editors]. New York: Le Jacq Publishing.

19. Chang L \& Drossman DA (2010) Rome Foundation Endpoints and Outcomes Conference 2009: optimizing clinical trials in FGID. Am J Gastroenterol 105, 722-730.

20. Passos MC, Lembo AJ, Conboy LA, et al. (2009) Adequate relief in a treatment trial with IBS patients: a prospective assessment. Am J Gastroenterol 104, 912-919.

21. EFSA Scientific Committee (2011) Statistical significance and biological relevance. EFSA J 9, 2372.

22. Withehead WE, Palsson OS, Levy RL, et al. (2006) Reports of "satisfactory relief" by IBS patients receiving usual medical care are confounded by baseline symptom severity and do not accurately reflect symptom improvement. Am J Gastroenterol 101, 1057-1065. 